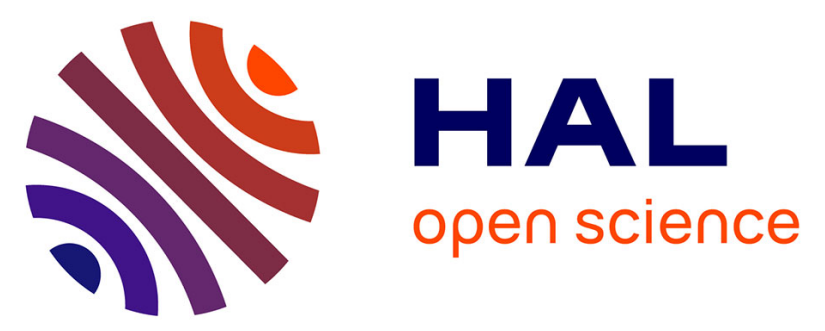

\title{
Tuning the morphology of functionalized silica using amphiphilic organosilanes
}

Romain Besnard, Guilhem Arrachart, Julien Cambedouzou, S Pellet-Rostaing

\section{To cite this version:}

Romain Besnard, Guilhem Arrachart, Julien Cambedouzou, S Pellet-Rostaing. Tuning the morphology of functionalized silica using amphiphilic organosilanes. Journal of Sol-Gel Science and Technology, 2017, 81 (2), pp.452-467. 10.1007/s10971-016-4202-1 . hal-01998488

\section{HAL Id: hal-01998488 \\ https://hal.science/hal-01998488}

Submitted on 28 Oct 2021

HAL is a multi-disciplinary open access archive for the deposit and dissemination of scientific research documents, whether they are published or not. The documents may come from teaching and research institutions in France or abroad, or from public or private research centers.
L'archive ouverte pluridisciplinaire HAL, est destinée au dépôt et à la diffusion de documents scientifiques de niveau recherche, publiés ou non, émanant des établissements d'enseignement et de recherche français ou étrangers, des laboratoires publics ou privés. 


\title{
Tuning the Morphology of Functionalized Silica using Amphiphilic Organosilanes.
}

\author{
Romain Besnard, Guilhem Arrachart,* Julien Cambedouzou,* Stéphane Pellet-Rostaing \\ Institut de Chimie Séparative de Marcoule, UMR 5257 CEA / CNRS / UM / ENSCM, BP17171, \\ F-30207 Bagnols-sur-Cèze, France. \\ E-mail : guilhem.arrachart@cea.fr $\quad T$ T : $33466791568 \quad$ F : 33466797611 \\ E-mail : julien.cambedouzou@enscm.fr__ T : $33466795803 \quad$ F : 33466797611
}

\begin{abstract}
The co-condensation of TEOS and an amphiphilic organosilane precursor containing an ammonium part is investigated in presence of different counter-ions. We highlight the morphological versatility offered by such "one pot" synthesis, resulting of the combinatory effects of the addition of TEOS and of the nature of the counter-ion involved in the sol-gel process. Indeed, the interactions at the interface governed by the ammonium species affect the shape of the aggregates, making of the counter-ion a critical morphological parameter. The morphology of the particles is also closely linked to the homogeneity of the system. Thereby, using the immiscibility of the TEOS/water mixture, we showed that it is possible to get macroporous, blackberry-like or cerasome materials. Oppositely, the use of tetrahydrofuran able to solubilize all the systems leads to monodisperse nanoparticles whose size can be modulated as a function of the involved counter ion.
\end{abstract}

\section{Keywords}

Organosilane / sol-gel / nanostructures / morphologies 


\section{Introduction}

Nanostructuring and control over morphology in hybrid solids are of great interest for the design of polyfunctional materials [1]. The sol-gel process is a powerful method for the production of solid hybrid materials. The funtionalization of silica with various organosilane compounds having amine was carried out via co-condensation, post-grafting of amino-silanes or surface hyperbranching polymerization [2]. Aminofunctionalized silica has been found to be useful for some base-catalyzed reactions, wastewater treatment, $\mathrm{CO}_{2}$ capture, further post-synthesis functionalization, and as platinum group metal, heavy metal and toxic oxyanion sorbents [3-8] to name a few. Multistep functionalization by attachment of different polyamines or dendrimers to pregrafted mesoporous substrates and surface growth of hyperbranched polyethyleneimine (PEI) is also described [9-10]. From the surfactant-mediated route, shape-controlled hybrids materials can be obtained. However some limitations, like the weak functionalization ratio and the non-homogeneous grafting due to blocking effects at the entrance of the pores, have been highlighted $[11,12]$.

Alternatively, hybrid silica can be prepared via the self-assembly of organosilane precursors bearing bridging organic unit that can be used to direct the structure of the solid network without addition of an external structuredirecting agent [13-15].

Self-structuring properties based on weak interactions between precursor molecules can influence the texture and morphology of materials after the sol-gel process [16]. An alternative procedure for functional silica materials with a high density of organic functions has been already described in a few studies, and consists in the use of amphiphilic organosilane molecules [17-21].

We recently demonstrated the amphiphilic abilities of amino-undecyl-triethoxysilane (AUT) when this molecule is associated with various acidic curvature agents [22]. We showed that the self-assembly is governed by the formation of ammonium species by the addition of an agent (in the form of a counter-ion) whose steric hindrance can introduce tunable curvature at the surface. Depending of the size of the curvature agent surrounding the amino part of the amphiphilic organosilane molecule, it has been possible to modulate the morphology in the form of lamellae, vesicular membranes or nanofibers.

Thanks to the siloxane part of the AUT, which can be hydrolyzed and condensed, such amphiphilic molecules can be engaged in a sol-gel process as for tetraethoxysilane (TEOS) silica precursors. The introduction of a silica precursor such as TEOS in the mixture implies that the siloxane part of both the AUT and the TEOS are likely to react and form Si-O-Si bridges. Therefore, the incorporation of TEOS in the organization may involve structural changes. In particular, a previous study devoted to the effect of TEOS on the self-assembly of AUT without excess of water demonstrated the possible reversibility of the self-assembly from lamellae to reverse cylindrical micelles, in which silica surrounds the organic core [23].

In this article, we explore the influence of the counter-ion on the morphology, the nanostructure and the composition of the final products. Starting from a system evolving in water, the aim of this study is to explore the combinatory effects of TEOS addition and of different curvature agents such as carbamic acid, hydrochloric acid, acetic acid, pivalic acid, and valproic acid on the structural arrangement of AUT during the sol-gel process. Then, the study was extended to the influence of the solvent on the morphological structure and the chemical composition 
of the solids. The mechanism of the structural evolution was followed by small angle X-ray scattering (SAXS) in order to highlight the incorporation effect of silica units in the resulting materials. As shown by Scanning Electron Microscopy (SEM) images, macroporous beads, blackberry-like particles, vesicles or nanoparticles can be obtained as function of the curvature agent used, which turns out to be a critical morphological parameter. Dynamic light scattering (DLS) and Fourier transform infra-red (FTIR) measurements reveal a consistency between the nanoparticle size and the curvature agent content in the material. The compositional study was completed by Raman spectroscopy and ${ }^{29} \mathrm{Si}$ solid state nuclear magnetic resonance (NMR) spectroscopy.

\section{Experimental section}

\subsection{Technical details}

SAXS experiments were performed on a set-up operating in transmission geometry. A Mo anode associated to a Fox2D multi-shell mirror (XENOCS) delivers a collimated beam of wavelength $0.710 \AA$. Two sets of scatterless slits [24] delimitate the beam to a square section of side length $0.8 \mathrm{~mm}$. A MAR345 imaging plate detector allows simultaneously recording scattering vectors $\mathbf{q}$ ranging from $0.25 \mathrm{~nm}^{-1}$ up to $25 \mathrm{~nm}^{-1}$. Samples were placed in glass capillaries of $2 \mathrm{~mm}$ diameter. Absolute intensities were obtained by measuring a calibration sample of high density polyethylene (Goodfellow) for which the absolute scattering was already determined. All SAXS profiles are plotted in log-log scale in order to highlight the dependency of the intensity versus a power law of the scattering vector $\mathbf{q}$.

High resolution transmission electron microscopy (HRTEM) was carried out at $200 \mathrm{kV}$ on a JEOL $2200 \mathrm{FS}$ microscope. Samples obtained after heat treatment were ground into a powder and dispersed in ethanol. Samples were deposited on 400-mesh carbon-coated copper grids. After ethanol evaporation, the grid was submitted to analysis.

Scanning electron microscopy (SEM) was performed on a FEI QUANTA 200 ESEM FEG operating at $15 \mathrm{kV}$ and equipped with a Everhart-Thornley detector or Large Field detector.

Wet scanning transmission electron microscopy (Wet-STEM) was performed on a FEI Quanta 200 ESEM FEG microscope operated at $30 \mathrm{keV}$. A Peltier stage and an inverse BSE detector are located below the final lens in the microscope chamber. A droplet of the diluted sample solution was deposited on a TEM copper grid coated with a holey carbon film. The grid was then placed on the Peltier stage previously cooled at $\mathrm{T}=2{ }^{\circ} \mathrm{C}$. In order to obtain a pure water vapor atmosphere in the ESEM chamber, an optimized pump down sequence was used to adjust the water partial pressure at $700 \mathrm{~Pa}: 12$ successive purges between $600 \mathrm{~Pa}$ and $1200 \mathrm{~Pa}$ lead to $99.5 \%$ water vapor in the ESEM chamber. Afterwards, the pressure is adjusted around $700 \mathrm{~Pa}$ to evaporate a very small amount of water from the droplet to adjust the water film thickness, allowing the observation of the sample through a water film.

DLS measurements were performed on an ALV-CGS3 with a red laser beam $(\lambda=638.2 \mathrm{~nm})$. The scattering angle was set to $173^{\circ}$. The temperature of the sample environment was controlled and stabilized at $20^{\circ} \mathrm{C}$. Sample quartz tubes (square glass cells) were cleaned-up with ethanol and then dried. The solutions were diluted to meet the requirements of turbidity and viscosity for DLS measurements. For DLS measurements, the error on Dh (hydrodynamic diameter) was principally due to the viscosity, the turbidity, the anisotropy and dispersity. DLS 
measurements were performed in triplicate. The PDI values (polydispersity indexes) reported in the Table S2 (see supporting information) illustrate the corresponding standard deviations.

Raman spectra were recorded on a Horiba Jobin Yvon LabRAM Aramis confocal Raman microscope, using an excitation wavelength of $\lambda=532 \mathrm{~nm}$ with a laser spot size of $\sim 1 \mu \mathrm{m}$, and with an objective of X50 long working distance. The incident laser power was kept lower than $0.8 \mathrm{~mW}$ in order to avoid any heating or structural sample damage.

Solid-state FT-IR spectra were obtained using an ATR crystal on a Perkin-Elmer 100 spectrometer at $4 \mathrm{~cm}^{-1}$ resolution.

The solid state ${ }^{29} \mathrm{Si}$ cross-polarization magic angle spinning (CP-MAS) NMR spectra were recorded on a Bruker 400 ultrashield VS spectrometer equipped with a $4 \mathrm{~mm}$ MAS probe. The MAS spinning rate was $12 \mathrm{kHz}$. The typical contact time was of 2-3 ms, and the typical recycle delay was 5-30 s.

\subsection{Synthesis of amino-undecyl-triethoxysilane}

The organic precursor was synthesized in three steps in anhydrous conditions. The synthesis was adapted from the one described in the literature [25]: Bromoundecene (Alpha Aesar) was first mixed with triethoxysilane and Karstedt catalyst both purchased from ABCR to obtain bromoundecyltriethoxysilane. After purification, the latter molecule was then used to form azidoundecyltriethoxysilane with sodium azide (Sigma Aldrich) into acetonitrile. Finally, the reduction of the azido group with Palladium (10\% Pd / C) from Sigma Aldrich into anhydrous ethanol under hydrogen atmosphere led to aminoundecyltriethoxysilane.

\subsection{Materials synthesis}

Primary amino groups are known for trapping carbon dioxide of air to lead to a carbamic acid. The pKa of the amine $(\mathrm{pKa}=10.6)$ allows the association of such a carbamic acid and a close amine to provide an ammonium carbamate dimer [26, 27]. The study of aminoundecyl-triethoxysilane in water has showed that this ability promotes the lamellar structural organisation of the resulting hybrid silica materials by forming ammonium carbamate bridges. Moreover, previous studies showed that the amphiphilic properties of dodecylamine with hydrochloric acid or carboxylic acids are governed by a proton transfer from the acid to the amine [28].

Taking into consideration these results, we choose here to investigate the effect of addition of TEOS in the mixtures containing different curvature agents: carbon dioxide, which forms a carbamic acid with the amine and leads to an ammonium carbamate dimer $\left(-\mathrm{NHCO}_{2} \cdot{ }^{+} \mathrm{H}_{3} \mathrm{~N}-\right)$, hydrochloric acid $(\mathrm{HCl})$ at two different concentrations, acetic acid $\left(\mathrm{CH}_{3} \mathrm{CO}_{2} \mathrm{H}\right)$, pivalic acid $\left(\left(\mathrm{CH}_{3}\right)_{3} \mathrm{CCO}_{2} \mathrm{H}\right)$, and valproic acid $\left(\left(\mathrm{CH}_{3} \mathrm{CH}_{2} \mathrm{CH}_{2}\right)_{2} \mathrm{CHCO}_{2} \mathrm{H}\right)$. These experiments have been performed in water and in THF, leading to 11 series of mixtures noticed $\mathrm{Sn}-\mathrm{X}$ series. S refers to the solvent ( $\mathrm{H}$ for $\mathrm{H}_{2} \mathrm{O}$, T for THF), $\mathrm{n}$ indexes refer to the molar ratios between TEOS and AUT, and finally X refers to the kind of agent used $\left(-\mathrm{CO}_{2}\right.$ for carbon dioxide, $-\mathrm{Cl}_{\mathrm{b}}$ for hydrochloric acid at $\mathrm{pH}=1.5,-\mathrm{Cl}_{\mathrm{a}}$ for hydrochloric acid at $\mathrm{pH}=0.86$, -Ac for acetic acid, -Piv for pivalic acid and -Val for valproic acid). These preparations are compared to a preparation without curvature agent, noted $\mathrm{Sn}$ series. The hydrolysis and the condensation reaction 
are performed by the combined action of water and of the respective agent, except for $\mathrm{H} 0-\mathrm{CO}_{2}$, where the hydrochloric acid is added for assuring the sol-gel reactions.

\subsubsection{Preparation of Hn-X series}

In order to prevent the pre-hydrolysis and the pre-organization of organosilane surfactants and TEOS for the sample prepared in water, our procedure first features the introduction of the AUT, secondly that of the respective amount of TEOS, and then the introduction of the curvature agent in a flask, except for the $\mathrm{Hn}-\mathrm{Cl}_{\mathrm{a}}$ and $\mathrm{Hn}-\mathrm{Cl}_{\mathrm{b}}$ series where the hydrochloric acid is added in the same time as the water. Then the samples are mixed for 60s in a vortex mixer. After the addition of solvent, the mixtures are placed in a thermostatic orbital shaker at $30{ }^{\circ} \mathrm{C}$ at 400 rpm for 3 days. Table 1 sums up the compositions of samples of the Hn-X series and Scheme 1 illustrates the methodology.

The total concentration of AUT plus that of TEOS is fixed according to equation (1).

$n_{\mathrm{H}_{2} \mathrm{O}}=500\left(n_{A U T}+n_{T E O S}\right)$

The concentration of the catalyst used for the sol-gel process is remained constant in order to keep equivalent catalytic activities in all the samples of the same Hn-X series.

Preparation of $\mathrm{Hn}, \mathrm{Hn}-\mathrm{Cl}_{a}$ and $\mathrm{Hn}-\mathrm{Cl}_{b}$

AUT and TEOS are placed in a flask and are mixed for $60 \mathrm{~s}$ in a vortex mixer. Then, $5 \mathrm{~mL}$ of water, hydrochloric aqueous solution of concentration $[\mathrm{HCl}]=0.138 \mathrm{~mol} . \mathrm{L}^{-1}$ or $[\mathrm{HCl}]=0.032 \mathrm{~mol} . \mathrm{L}^{-1}$ are respectively added for $\mathrm{Hn}, \mathrm{Hn}$ $\mathrm{Cl}_{\mathrm{a}}$ and $\mathrm{Hn}-\mathrm{Cl}_{\mathrm{b}}$

\section{Preparation of $\mathrm{Hn}-\mathrm{CO}_{2}$}

AUT and TEOS are placed in a flask and are mixed for 60s in a vortex mixer. The resulting oil is placed under $\mathrm{CO}_{2}$ flow during $15 \mathrm{~min}$. The formation of a gel is observed immediately. Then, $5 \mathrm{~mL}$ of a hydrochloric aqueous solution at $\mathrm{pH}=1.5$ are added.

\section{Preparation of Hn-Ac, Hn-Piv and Hn-Val series}

AUT and TEOS are placed in a flask and are mixed for $60 \mathrm{~s}$ in a vortex mixer. Then, the acetic acid, pivalic acid or valproic acid is respectively added for $\mathrm{Hn}-\mathrm{Ac}, \mathrm{Hn}-\mathrm{Piv}$ and $\mathrm{Hn}-\mathrm{Val}$. In the three cases, the mixture stays oily and homogeneous. Then, $5 \mathrm{~mL}$ of water are added.

\subsubsection{Preparation of $\mathbf{T n}-\mathrm{X}$ series}

The use of THF as solvent allows the solubilisation of all the introduced components. The procedure consists in the solubilisation of the AUT in THF, followed by addition of the respective amount of TEOS, and then that of the curvature agent and finally of the stoichiometric amount of water required for the hydrolysis and condensation 
of the siloxane part of the organosilane surfactant and TEOS. One water molecule is used in order to consider the hydrolysis and condensation of two siloxane groups (Et-O-Si-). Thereby, the water content is calculated as follows:

$n_{\mathrm{H}_{2} \mathrm{O}}=1.5 n_{A U T}+2 n_{T E O S}$

The total concentration of AUT plus that of TEOS is fixed aiming at having an equivalent concentration than in the experiment made in water as solvent. According to the molecular mass and density of the THF compared to those of water, the THF content in the mixture can be calculated as follows:

$n_{T H F}=112.5\left(n_{A U T}+n_{T E O S}\right)$

Table 2 sums up the compositions of samples of the Tn-X series and scheme 2 illustrates the methodology.

Preparation of $T n, T n-C l_{a}$ and $T n-C l_{b}$ series

AUT and TEOS are placed in a flask containing the THF. The resulting solutions are mixed for 60s in a vortex mixer. Then, the catalytic amount of water, hydrochloric aqueous solution at $\mathrm{pH}=0.86$, or hydrochloric aqueous solution at $\mathrm{pH}=1.5$ is repectivelyadded for $\mathrm{Tn}, \mathrm{Tn}-\mathrm{Cl}_{\mathrm{a}}$ and $\mathrm{Tn}-\mathrm{Cl}_{\mathrm{b}}$ series.

Preparation of $\mathrm{Tn}-\mathrm{CO}_{2}$ series

AUT and TEOS are placed in a flask containing the THF. The resulting solutions are mixed for $60 \mathrm{~s}$ in a vortex mixer prior to be placed under $\mathrm{CO}_{2}$ flow during $15 \mathrm{~min}$. Then, the catalytic amount of hydrochloric aqueous solutions at $\mathrm{pH}=1.5$ is added.

Preparation of Tn-Ac, Tn-Piv and Tn-Val series

AUT and TEOS are placed in a flask containing the THF. The resulting solutions are mixed for $60 \mathrm{~s}$ in a vortex mixer. Then, the acetic acid, pivalic acid, or valproic acid is respectively added for Tn-Ac, Tn-Piv and Tn-Val. Then, the catalytic amount of water is added to the homogeneous solution.

\section{Results and discussion}

\subsection{Systems evolving in aqueous phase: $\mathrm{Hn}-\mathrm{X}$ series}

\subsubsection{Structure and morphology}

The first experiments were performed in water aiming at determining the role of the addition of TEOS in the organization process and in the morphology of materials. We observed different behaviors as function of the catalytic effect or amount of TEOS involved in the sol-gel reaction. Changes from suspension to gel, clear suspension to powder or no significant evolution were indeed observed (Table S1 see supporting information).

The addition of TEOS in the starting mixtures seems to change the aspect and the characteristics of the samples for several series but not for all of them. The structural effects due to the contribution of such silica units into the initial structure were revealed by SAXS experiments. The SAXS profile of the H0 material (Fig 1) was previously discussed in our former study [22]. The positions of the four structure peaks testify to a lamellar structure featuring 
stacked bilayers. The hypothesis according to which TEOS does not affect the organization would imply that the condensation of TEOS and the siloxane part of the AUT molecules would occur separately. Such hypothesis would give a SAXS profile which would not present important changes compared to the initial lamellar structure obtained without TEOS.

\section{Hn series: no curvating agent}

In the present instance, the addition of TEOS in the mixture seems to lead to important changes in the organization (Fig 1). Indeed, the SAXS patterns of H0.5, H1 and H1.5 materials (dash, dots and rings) exhibit wide structure peaks whose positions are close to those related to the lamellar structure of the $\mathrm{H} 0$ material. The shift of the second peak, from $3.65 \mathrm{~nm}^{-1}$ (dots) to $3.3 \mathrm{~nm}^{-1}$ when $\mathrm{n}$ increases, involves a slight swelling of the structure. The lattice parameter of the lamellar structure was evaluated at $4 \AA$ for $\mathrm{H} 1.5$ material, which is in agreement with the insertion of the fragment $\left(\mathrm{Si}_{-}-\mathrm{O}_{2}-\right)_{2}$ into the silica wall of the bilayers.

By assuming that the TEOS and siloxane part condense jointly, the silica units coming from the condensation of TEOS are localized in the silica walls of the bilayers. The peak width suggests that the distribution of silica units along the bilayer is non homogeneous, leading to a swelling but also a distortion of the structure. As the TEOS ratio increases in the composition, the structure peaks become wider until complete merging between the first and the second one (H5).

Therefore, the insertion of silica units coming from the condensation of TEOS generates significant structural modifications. The TEOS addition also affects the morphology, as revealed by SEM images (Fig 2). Starting from a powder that does not present any particular morphology (Fig 2a), the addition of TEOS seems to bring a macroporosity in the final material (Fig $2 \mathrm{~b}, 2 \mathrm{c}$ ).

The H1 material is composed of different kinds of objects. Indeed, we observed macroporous beads which are isolated or merged (Fig S1a). The bead size is included between 10 and $100 \mu \mathrm{m}$. The pores seem to be connected and their diameters were evaluated at 1 to $2 \mu \mathrm{m}$ according to the SEM images. Other beads are completely empty forming hollow spheres (Fig S1b), or are macroporous planar objects, which can be due to the vial wall effects during the sol-gel process (Fig S1c). The hydrophobic character of TEOS does not allow it to be miscible with water. In basic condition, as the present case, the condensation reaction is favored compared to the hydrolysis reaction. The formation mechanism of hollow spheres could be due to the hole left by the condensation of TEOS droplets as already described [29]. The formation of such hollow spheres and also the formation of macroporous beads could also be the consequence of a double emulsion, where water droplets would be trapped inside TEOS droplets. Both sorts of droplets would be stabilized by the AUT molecules at the interface between water and TEOS. By a slow hydrolysis process and a fast condensation process, the silica precursor cannot be solubilized in the solution and the emulsion is fixed and "fossilized". The insertion of more TEOS into the mixture leads to large walls separating the pores (Fig 2c). However, the low AUT ratio in the mixture does not permit to stabilize many droplets, leading to a bi-continuous media.

Although a part of the porosity seems to be opened, another part is closed and thus unavailable for gas adsorption. This was highlighted by $\mathrm{N}_{2}$ adsorption/desorption measurements with small amounts of adsorbed nitrogen measured for the materials, therefore indicating a closed structure. 
The amino function can be trapped inside the silica matrix or localized at the surface. In order to check the mechanism involved, we performed an experiment aiming at locating the amino functions of the AUT molecule in the $\mathrm{H} 1$ material. The use of gold nanoparticles (gold $\mathrm{Np}$ ) for locating amino groups has been already described [30]. The difference of affinity between the amino and the silanol function for gold Np allows determining their respective location. Indeed, gold $\mathrm{Np}$ can be trapped at the surface by the amines but not by the silanol groups at the surface of silica.

The white dapples in the SEM images of the H1 and H5 materials of the Fig S2 (see supporting information) indicate the location of the gold $\mathrm{Np}$ and furthermore the position of the amino functions. The areas connecting the fossilized droplets and also the walls of droplets are covered of relatively well dispersed gold Np, involving a good dispersion of the amino functions at the surface of the material. Therefore, this observation favors the second hypothesis, consisting in a double emulsion where water droplets are trapped in TEOS droplets with regards to the first hypothesis involving holes left by the condensation of TEOS droplets. A schematic illustration of the first mechanism is proposed in Fig 3.

It appears that the addition of TEOS acts on the self-assembly of the AUT molecules by swelling the structure. In addition, its hydrophobic character before hydrolysis highlights the amphiphilic properties of the AUT molecule, leading to the formation of porous materials.

\section{$\mathrm{Hn}-\mathrm{CO}_{2}$ series}

Aiming at determining the bending effects, we extend the study by exploring the structural and morphological effects on mixtures containing curvature agents. First, the use of carbon dioxide was studied with different TEOS ratios in the mixtures leading to $\mathrm{H} 0-\mathrm{CO}_{2}, \mathrm{H} 1-\mathrm{CO}_{2}$ and $\mathrm{H} 5-\mathrm{CO}_{2}$ materials. The structural evolution was similar to the evolution observed with materials prepared without curvature agent (Fig S3). However, the porosity produced by the fossilization of the emulsion in the $\mathrm{H} 1$ and $\mathrm{H} 5$ materials is completely absent in the $\mathrm{H} 1-\mathrm{CO}_{2}$ and $\mathrm{H} 5-\mathrm{CO}_{2}$ materials (Fig S4). Indeed, the materials are composed of dense fragments of nonspecific morphology.

The bubbling of carbon dioxide generates carbamic acid species by reacting with the amine of the AUT molecule in the solutions. Then, the attractive interaction between carbamic acid species and free amine of AUT molecule thereby produces ammonium carbamate dimers [20]. This phenomenon could be the origin of the collapsing and the destabilization of the water/TEOS emulsion observed in H1 and H5. Moreover, in this case the sol-gel process is enhanced by an acidic catalyst involving a fast hydrolysis reaction compared to the condensation. The large amount of ethanol could also destabilize the emulsion.

\section{Hn-Clb series}

The hydrochloric acid was then experimented as curvature agent in different conditions. The concentration of hydrochloric acid remains constant while the ratio AUT/TEOS changes. Therefore, when the ratio HCl/AUT increases, the $\mathrm{pH}$ decreases. The ratio $\mathrm{HCl} / \mathrm{AUT}$ was smaller than $1 \mathrm{for} \mathrm{H} 0, \mathrm{H} 0.5, \mathrm{H} 1, \mathrm{H} 1.5$. The mixtures were considered to be in basic condition $(10<\mathrm{pH}<11)$ taking into account the respective concentration and $\mathrm{pKa}$ of the AUT. For $\mathrm{HCl} / \mathrm{AUT}$ ratios higher than 1 (for $\mathrm{H} 5$ and $\mathrm{H} 10$ ), the $\mathrm{pH}$ was estimated to 2.15 and 1.7, respectively. 
The structural study reveals the same evolution than the experiment without curvature agent (Fig S5). The initial lamellar structure was first swelled and amorphized. Nevertheless, many changes have been observed in the sample morphology. The surface of blackberry-like particles formed from mixture without TEOS (Fig 4a) seems to be progressively smoothed by the addition of TEOS. Indeed, Fig $4 \mathrm{~b}$ highlights particles exhibiting traces of the blackberry-like morphology. The silica units coming from TEOS condensation swell the structure and fill the interstices and cavities left by the initial organization, leading to filled spherical particles whose size distribution is included between $500 \mathrm{~nm}$ and $10 \mu \mathrm{m}$ (Fig 4c). Although, despite the pH conditions changes, the morphology of H5 was almost identical, excepted that the small particles seem to be more abundant (inset Fig 4d). This sample has an intermediate morphology between the filled beads prepared in basic conditions (Fig 4c) and the collapsed vesicles observed for H10 (Fig 4e).

Considering that the $\mathrm{H} 0-\mathrm{Cl}_{\mathrm{b}}, \mathrm{H} 0.5-\mathrm{Cl}_{\mathrm{b}}$ and $\mathrm{H} 1-\mathrm{Cl}_{\mathrm{b}}$ were prepared with a default of hydrochloric acid, some amines are protonated while others remain free. Therefore, by the same phenomena described for $\mathrm{H} 1-\mathrm{CO}_{2}$ and $\mathrm{H} 5-\mathrm{CO}_{2}$ materials, this morphology is probably due to attractive interactions between ammonium chloride and amine species, which lead to dense particles. By decreasing the AUT ratio in favor of TEOS, the free amine species fraction decreases until they are all protonated, annihilating the attractive interactions. The interactions at the interface between two bilayers then become repulsive and lead to the formation of vesicles.

Raman microspectra of individual microscopic beads were recorded in different regions of the material, allowing us to check the homogeneity of the TEOS condensation inside the material. As exhibited in Fig S6, the Raman spectra of $\mathrm{H} 0-\mathrm{Cl}_{\mathrm{b}}, \mathrm{H} 1-\mathrm{Cl}_{\mathrm{b}}$ and $\mathrm{H} 5-\mathrm{Cl}_{\mathrm{b}}$ exhibit bands at 1063,1297 and $1440 \mathrm{~cm}^{-1}$ respectively featuring the C-C stretching mode, the $\mathrm{CH}_{2}$ twisting, and $\mathrm{CH}_{2}$ bending modes of the organic molecule. These bands are still observed even when the amount of TEOS is important in regards to the organic part of AUT, as can be seen for $\mathrm{H} 5-\mathrm{Cl}_{\mathrm{b}}$.

The most notable discrepancies are the increase of the band at $500 \mathrm{~cm}^{-1}$ and of the band at $1030 \mathrm{~cm}^{-1}$ as the TEOS ratio increases, resulting of the larger silica contribution. Similar spectra were obtained in different regions of the materials, suggesting that the silica units are condensed homogeneously in the material.

\section{$\mathrm{Hn}-\mathrm{Cl}_{\mathbf{a}}$ and $\mathrm{Hn}-\mathrm{Ac}$ series}

The observation of collapsed vesicles for $\mathrm{H} 10-\mathrm{Cl}_{\mathrm{b}}$ - which is prepared in acidic conditions - is in agreement with the vesicles observed in the $\mathrm{H} 0-\mathrm{Cl}_{\mathrm{a}}$ solutions previously studied in a former article [22].

The $\mathrm{H} 1-\mathrm{Cl}_{\mathrm{a}}$ and $\mathrm{H} 5-\mathrm{Cl}_{\mathrm{a}} \mathrm{SAXS}$ profiles were compared to that of $\mathrm{H} 0-\mathrm{Cl}_{\mathrm{a}}(\mathrm{Fig} 5)$.

The form factor does not change drastically with the TEOS ratio. Planar objects are thus also formed owing to the $\mathrm{q}^{-2}$ power law followed by the intensity. Taking into consideration the observations previously made on $\mathrm{H} 0-\mathrm{Cl}_{\mathrm{a}}$ and $\mathrm{H} 10-\mathrm{Cl}_{\mathrm{b}}$, it also suggests that the objects contained in the $\mathrm{H} 1-\mathrm{Cl}_{\mathrm{a}}$ and the $\mathrm{H} 5-\mathrm{Cl}_{\mathrm{a}}$ solutions are vesicles. This hypothesis was confirmed by images performed by Wet-STEM (Fig 6). Nevertheless, the higher intensity in the SAXS profiles suggests that different possibilities can be considered as function of the condition used: the vesicles can be larger, more abundant, or the molecular density in the aggregates could be larger.

Therefore, DLS experiments were conducted. The results indicate the presence of objects slightly larger (Table $\mathrm{S} 2)$ for $\mathrm{H} 5-\mathrm{Cl}_{\mathrm{a}}(\sim 170 \mathrm{~nm})$ than those contained in $\mathrm{H} 0-\mathrm{Cl}_{\mathrm{a}}(\sim 130 \mathrm{~nm})$. The difference in size is not significant, 
indicating that the three possibilities remain reliable. The structure peak at $0.8 \mathrm{~nm}^{-1}$ characterizing the interactions between the membranes in $\mathrm{H} 0-\mathrm{Cl}_{\mathrm{a}}$ suspensions is missing in the SAXS profile of the $\mathrm{H} 1-\mathrm{Cl}_{\mathrm{a}}$ and of the $\mathrm{H} 5-\mathrm{Cl}_{\mathrm{a}}$ solutions, involving that these interactions are absent. Therefore, the insertion of TEOS does not seem to contribute to the organization of $\mathrm{Hn}-\mathrm{Cl}_{\mathrm{a}}$ series in regards to the initial vesicular aggregation. The main effect is the interconnection of the vesicles and the high viscosity of the suspension.

Comparatively, for the materials prepared by using acetic acid as curvature agent instead of hydrochloric acid, the SAXS profile evolution of solutions is similar (Fig $5 b$ ). The intensity increases and follows a $q^{-2}$ power law as the TEOS ratio increases, featuring the presence of planar objects. No gel formation was observed for these samples even after four months. As observed for its analogous prepared with hydrochloric acid, the Wet-STEM images (Fig 6b) show vesicles. However, micelles of different sizes were observed on the images. DLS measurements confirm the presence of the two larger object sizes, with a polydispersity index of 0.2 involving a restricted distribution around the size values (Table S2).

\section{Hn-Piv and Hn-Val series}

Contrarily to the solutions prepared with hydrochloric acid and acetic acid, those prepared with pivalic acid do not lead to a suspension of vesicles when TEOS is added in the mixture. From one equivalent of TEOS by AUT molecule, the preparation H1-Piv leads to a precipitate. The SAXS pattern of such a material (Fig 7a) shows two broad lumps whose positions $\left(2 \mathrm{~nm}^{-1}\right.$ and $4 \mathrm{~nm}^{-1}$ ) could feature a distorted lamellar structure. Then, the presence of more TEOS in the composition in H5-Piv generates structural evolution. The intensity follows a $\mathrm{q}^{-4}$ power law, attesting of sharp interfaces between the aggregates and the surrounding medium. A structure factor was also observed at around 2 to $3 \mathrm{~nm}^{-1}$ featuring interactions at a mesoscopic range; the corresponding typical distances are comprised between once or twice the fully extended AUT molecule length.

The initial structure and morphology of materials prepared with pivalic acid are very different than those prepared with valproic acid. Indeed, as described in a precedent article [22], the H0-Piv is a vesicular suspension of cerasomes, and the H0-Val material is a soft and stretchable solid composed of core-shell organosilica nanofibers organized in a 2D hexagonal structure. However, the structural evolutions seem to be identical for both Piv and Val based materials as the TEOS ratio increases (Fig 7).

The SEM images indicate that large spherical particles of size 10-50 $\mu \mathrm{m}$ were obtained for the H5-Piv material (Fig S7a), while smaller objects without particular morphology were obtained for H5-Val (Fig S7b).

\subsubsection{Chemical composition of the solids}

IR spectroscopy has been applied to investigate the composition of materials. Indeed, the analysis of IR spectra provides an important indication of the influence of the TEOS ratio inside the mixtures. The IR spectrum of H0 (Fig S8) features seven main absorption bands. The two firsts at 2920 and $2850 \mathrm{~cm}^{-1}$ are respectively characteristic of $\mathrm{CH}_{2}$ antisymmetric and symmetric stretching motions of the AUT. Then, the weak band at $1590 \mathrm{~cm}^{-1}$ results of the stretching vibration mode of the carbamate species due to the ability of the amino function to catch the carbon dioxide from air and water [31]. The bands located at $1470 \mathrm{~cm}^{-1}$ and $1100 \mathrm{~cm}^{-1}$ were respectively attributed to the 
$\mathrm{C}-\mathrm{H}$ bending mode and $\mathrm{C}-\mathrm{C}$ stretching vibration mode of the alkyl chain. The bands at $1100,1050 \mathrm{~cm}^{-1}$ and 930 $\mathrm{cm}^{-1}$ are assigned to the $\mathrm{Si}-\mathrm{O}(-\mathrm{Si})$ and $\mathrm{Si}-\mathrm{OH}$ stretching modes [32].

By comparing the transmission of the bands corresponding to the $\mathrm{CH}_{2}$ antisymmetric stretching mode and the band characteristic of the Si-O stretching mode in the different compositions as $\mathrm{n}$ increases, it appears possible to appreciate the ratio between the organic contribution (undecyl chain of AUT) and the silica contributions coming from the condensation of siloxane part of AUT and TEOS (Fig S8).

As the $\mathrm{Si}-\mathrm{O}$ contribution is convoluted with the $\mathrm{C}-\mathrm{C}$ stretching vibration mode of the alkyl chain and the potential residual ethoxy group $\left(\mathrm{CH}_{3} \mathrm{CH}_{2} \mathrm{OSi}\right)\left(1100 \mathrm{~cm}^{-1}\right)$, the FTIR analysis does not constitute a quantitative measurement. However, the ratio value $\tau$ described by the equation 4 was proposed in order to evaluate the ratio of silica units compared to the organic molecule into the final materials.

$\tau=\frac{\left(T_{0}-T_{S i-O}\right)}{\left(T_{0}-T_{\mathrm{C}-\mathrm{H}}\right)}$

where $T_{0}, T_{S i-O}$ and $T_{C-H}$ are the transmitted intensity of the baseline, of the bands characteristic of the Si-O stretching mode and of the $\mathrm{C}-\mathrm{H}$ antisymmetric stretching mode, respectively. Fig 8 gives the ratios $\tau$ for the compositions leading to materials. The results exhibit a clear increase of the Si-O contribution as the TEOS ratio increases inside the mixture for all the materials.

The calculation of these ratios demonstrates that $\tau$ seems to follow a linear law for all the materials (from 0 to 5 TEOS), where the slope qualitatively measures the ability of the system to incorporate TEOS in the material. The ratios of the different contributions of materials are proportional to the TEOS content of the initial mixture. The slopes and the $\mathrm{R}^{2}$ of the regressions are reported in Table S3. The slope values of the different systems indicate that the hydrochloric acid and the valproic acid seem to improve moderately the incorporation of TEOS in the material (respective slope value of 0.85 and 0.98), in comparison to $\mathrm{CO}_{2}$, which exhibits the smaller slope value (0.66). However, the slope value of the Hn-Val series has to be handled carefully considering that the intensity of the $\mathrm{C}-\mathrm{H}$ antisymmetric vibration mode can be affected by the $\mathrm{C}-\mathrm{H}$ vibration and the $\mathrm{OH}$ vibration of the valproic acid itself. The FTIR analysis testifies also to the presence of ammonium carboxylate species through the large band located at $1540 \mathrm{~cm}^{-1}$ as revealed for H0-Val, H1-Val and H5-Val in Fig S9.

The conclusions reached by the proposed model based on FTIR spectroscopy were then completed by ${ }^{29} \mathrm{Si}$ solid state NMR. The silica matrix composition and the AUT/TEOS ratios were highlighted by ${ }^{29} \mathrm{Si}$ solid state NMR measurements (Fig S10). This technique allows discriminating the nature of silicon atoms (those coming from the siloxane part of the AUT and TEOS), and also their condensation state. The percentage of the different $\mathrm{T}^{\mathrm{x}}$ and $\mathrm{Q}^{\mathrm{x}}$ substructures featuring the $\mathrm{R}-\mathrm{C}-\mathrm{Si}(\mathrm{OSi}) \mathrm{xOH}_{3-\mathrm{x}}$ and the $\mathrm{Si}(\mathrm{OSi}) \mathrm{xOH}_{4-\mathrm{x}}$ units respectively in the materials are obtained by the deconvolution of the spectra (Fig S11) and are reported in Table S4. The spectrum of H0 exhibits two peaks at -59 and $-67 \mathrm{ppm}$ corresponding to $\mathrm{T}^{2}$ and $\mathrm{T}^{3}$ units, reflecting a relatively high condensation rate. The $\mathrm{Si}-\mathrm{C}$ bond is retained during the sol-gel reaction since only $\mathrm{T}^{\mathrm{x}}$ units were resolved. No resonance attributable to $\mathrm{SiO}_{2}$ groups (Q units) was observed for this material. The spectra of $\mathrm{H} 1$ and $\mathrm{H} 5$ exhibit two supplemental peaks at -99 and $-109 \mathrm{ppm}$ attributed to $\mathrm{Q}^{3}$ and $\mathrm{Q}^{4}$ units. These peaks are related to the introduction of TEOS in the medium, and reveal a satisfying condensation of TEOS. In addition, the calculated ratios $\mathrm{Q}^{\mathrm{x}} / \mathrm{T}^{\mathrm{x}}$ confirm a composition close to the TEOS/AUT ratios introduced in the initial mixtures (Table S4). We can also note that no 
signal is visible at $-47 \mathrm{ppm}$ and $-82 \mathrm{ppm}$, involving that no remnant unhydrolyzed monomers are present in the materials [33]. The ${ }^{29} \mathrm{Si}$ solid state NMR results of the $\mathrm{H} 5-\mathrm{CO}_{2}, \mathrm{H} 5-\mathrm{Cl}_{\mathrm{b}}$, H5-Piv, and H5-Val materials exhibit comparable spectra than H5 (Fig S12) with mainly $\mathrm{T}^{3}, \mathrm{Q}^{3}$ and $\mathrm{Q}^{4}$ units, involving that whatever the catalyst engaged in the preparation of the materials, the sol-gel process is well conducted and lead to a dense silica matrix. From the different analyses, it appears that the introduction of TEOS seems to act in the self-assembly by reacting with the siloxane part of the AUT molecules and this regardless of the amount of TEOS introduced or of the nature of the curvature agent involved during the sol-gel process. Indeed, the structural study showed that TEOS seems to act in the self-assembly by incorporating and swelling the structures. Although the curvature agent does not affect drastically the quantitative introduction of silica units in the material, the interactions at the interface governed by the ammonium species affect the shape of aggregates, therefore demonstrating that the curvature agent acts as a critical morphological parameter.

In this system, the formation of an emulsion due to the immiscibility of TEOS in water could be responsible for a large size distribution of objects in the resulting material. Furthermore the formation of fossilized emulsion suggested by the morphology of the particles could be a direct consequence of this immiscibility.

In order to support this statement, it appears interesting to check whether, in a system without formation of emulsion, if the sol-gel process leads to homogeneous materials in morphology and size. For this purpose, and aiming at getting free from the problem of immiscibility of TEOS and water, a similar study was performed in tetrahydrofuran (THF).

\subsection{Systems evolving in THF: $T n-X$ series}

The same experiment was thus reproduced with other organic solvents aiming at determining their roles in the organization process and in the morphology of materials. THF was selected for its weak polarity. Oppositely to the experiments performed in aqueous solution, all the Tn-X samples lead to white powders materials except T0Piv and T0-Val, which lead to clear soft materials. The study of the solvent effects on the chemical composition, the structure and the morphology of the final materials was conducted in the same manner as previously described.

\subsubsection{Structure and morphology}

The structural evolution is similar to that observed in aqueous solutions as TEOS content increases. A swelling and an amorphization of the lamellar structures is observed (Fig S13).

\section{Tn series}

Beyond the similarities with the Hn series, Fig 9 illustrates morphological changes in the Tn series. Indeed, the T0 material prepared in THF without TEOS first appears to be composed of planar nanoparticles stacked on top of each other (Fig 9a), while the H0 analogous material prepared in water does not exhibit any specific morphology. In addition, instead of generating porosity, the introduction of TEOS in the composition seems to grow and connect the initial planar objects, making them spherical first (Fig 9b), and then elongated (Fig 9c). The trend of the AUT molecules to be aligned probably influences the growth along one direction. 
Owing to the polarity of the THF solvent, these morphological differences could be explained by the absence of the TEOS/THF emulsion. Therefore, the AUT molecule, the TEOS and the catalytic amount of water are totally solubilized, leading to particle sizes relatively monodisperse. A similar phenomenon leading to connected aggregates was observed in pentane, which is also able to solubilize the TEOS and the AUT molecule before hydrolysis. However, the particle morphologies are less defined and the distribution of particle sizes is larger (Fig S14). The introduction of the catalytic amount of water is significant enough for the formation of an emulsion in pentane. As the hydrolysis and condensation reactions occur, the water is consumed and the emulsion disappears, leading to collapsed aggregates.

Insofar THF seems to meet most of the requirements sought for good solvent to prepare homogeneous materials, we focus our study on the exploration of the curvature agent effects in THF.

\section{Tn-CO2 series}

As expected, the bubbling of $\mathrm{CO}_{2}$ gas into the solution does not affect the structural evolution as the TEOS ratio increases as shown in SAXS measurements (Fig S15). However, in an opposite way to the Tn series materials, the particle size of Tn- $\mathrm{CO}_{2}$ series materials decreases with the addition of TEOS. Starting from large merged spherical particles of size around $30 \mu \mathrm{m}$ for $\mathrm{T} 0-\mathrm{CO}_{2}$ material (Fig 10a), the particles become smaller with only one equivalent of TEOS in T1-CO $\mathrm{CO}_{2}$ (Fig 10b), until reaching 50-100 nm for $\mathrm{T} 5-\mathrm{CO}_{2}$ (Fig 10c). Such a particular morphology is probably inherent to the use of carbon dioxide, which reacts with the amine of the AUT molecule. The carbamic acid therein formed at the interface between the bilayers could give sufficient interactions for a packing on large distances.

The addition of silica units inside the bilayers involves a swelling of the structure which introduces a bending at the surface. The main difference between the preparation of $\mathrm{Tn}$ and $\mathrm{Tn}-\mathrm{CO}_{2}$ series is the kind of catalysis used for the sol-gel process. Indeed, the preparation of the first one is enhanced by the association of the amine of the AUT molecule and water, while that of the second series is enhanced by a catalytic amount of hydrochloric acid. We therefore suggest that the hydrochloric acid was responsible of the reversal growth.

\section{Tn-Clb series}

The use of hydrochloric acid without carbon dioxide validates this hypothesis through the $\mathrm{Tn}-\mathrm{Cl}_{\mathrm{b}}$ series. The difference between the preparation of the $\mathrm{Tn}$ and $\mathrm{Tn}-\mathrm{Cl}_{\mathrm{b}}$ series is only the sub-stoichiometric amount of hydrochloric acid. The difference between the preparation of $\mathrm{Tn}-\mathrm{CO}_{2}$ and $\mathrm{Tn}-\mathrm{Cl}_{\mathrm{b}}$ series is the ammonium species (carbamate or chloride). These weak changes in the composition seem to bring critical morphological differences compared to the Tn series but not in regards of the $\mathrm{Tn}-\mathrm{CO}_{2}$ series. Indeed, while the T5 materials was composed of elongated objects possessing a section of about $400 \mathrm{~nm}$, the $\mathrm{SEM}$ images of the $\mathrm{T} 5-\mathrm{Cl}_{\mathrm{b}}$ material exhibit nanoparticles (Fig 11a) similar to those observed for $\mathrm{T} 5-\mathrm{CO}_{2}(50-100 \mathrm{~nm})$. The inset in Fig 11a also reveals a very well organized packing, which can be explained by the interconnection of the nanoparticles as seen from the TEM analysis (Fig 11b).

\section{Tn-Ac, Tn-Piv and Tn-Val series}


The morphological effect of other curvature agents such as acetic acid, pivalic acid and valproic acid has also been explored. The T5-Ac, T5-Piv, and T5-Val materials are prepared with 4.1 equivalents of corresponding acid per AUT molecule in order to maintain a constant acid concentration in all series. Even if the number of curvature agent equivalents is not comparable between $\mathrm{T} 5-\mathrm{Cl}_{\mathrm{b}}$ and the other samples, we note some similarities concerning the morphology (Fig 12). The SAXS profiles of T5-Clb, T5-Ac, T5-Piv, and T5-Val are all almost identical (Fig S16) indicating a similar swelled meso-structure. The SEM images of these materials reveal the formation of nanoparticles. However, the particle shape of T5-Ac and T5-Val materials looks spherical and non-connected (Fig $12 \mathrm{a} \& 12 \mathrm{c}$ ), while that of T5-Piv is less easy to determine (Fig 12b).

In order to get more information on the size distribution of particles, we performed DLS measurements. The dispersion of the particles of the different materials was not easy. The resulting DLS correlogramm of the suspensions did not lead to relevant interpretations due to the strong interactions that seem to stick the particles together. For that purpose, we performed DLS measurements directly from the supernatants of the fresh suspensions from the different materials. Ethanol was added to meet the requirements of turbidity and viscosity. The measurements of $\mathrm{T} 5$ and $\mathrm{T} 5-\mathrm{CO}_{2}$ suspensions were unfortunately not interpretable. The results reported in Table S5 are in agreement with the typical distances observed in the SEM images. Both analysis methods converge toward the same conclusion: the use of carboxylic acid allows increasing the particles sizes compared to the hydrochloric acid. Furthermore, we also observed a size difference for T5-Piv and T5-Val compared to T5-Ac, involving that the particle size does not only depend on the catalytic activity but also on the curvature agent size.

\subsubsection{Chemical composition of the solids}

Depending on the TEOS content in the initial mixture, FTIR spectroscopy shows that the final material composition follows a distinct evolution than the one observed for materials prepared in aqueous solution. The $\tau$ values evolution featuring the ratio between the organic contribution (undecyl chain of AUT and organic catalyst) and the silica contribution (coming from the condensation of the siloxane part of AUT and TEOS) are showed in Fig 13a.

Contrarily to the materials prepared in water, the $\tau$ values of most of the materials prepared in THF do not follow a linear law as the TEOS increases. The materials series prepared without curvature agent and with $\mathrm{CO}_{2}$ do not exhibit linear evolutions as the TEOS content increases. The $\mathrm{T} 1-\mathrm{CO}_{2}$ material exhibits a $\tau$ value of 4.1 , signifying an important silica unit content coming from TEOS in the material. Then this trend seems to be attenuated for T5$\mathrm{CO}_{2}$.

This observation suggests that the condensation kinetic of TEOS is favored in comparison to that of AUT and the ratio meets an equilibrium. Owing to the ability of the primary amine function of the AUT molecule to trap $\mathrm{CO}_{2}$ from air, it is not surprising to observe the same phenomenon for the Tn series. Nevertheless, the equilibration does not seem to be reached.

The compositions containing the hydrochloric acid leads to materials whose evolution can be simulated by a straight line with $\mathrm{R}^{2}$ close to 1 (Table S6). The slope values differ according to the curvature agents. Therein, we highlight the more important content of silica units coming from TEOS for $\mathrm{Tn}-\mathrm{Cl}_{\mathrm{b}}$ series than for the others. 
Otherwise, provided that the slope of the $\mathrm{Tn}-\mathrm{Cl}_{\mathrm{b}}$ series is more important than that of the homologous material prepared in water $\mathrm{Hn}-\mathrm{Cl}_{\mathrm{b}}$, we can assume that the THF and the corresponding homogeneous media favor the condensation of TEOS in regards to the AUT molecules with hydrochloric acid.

The acetic acid seems to favor the condensation of AUT molecule compared to the TEOS molecule. As for TnPiv and Tn-Val series, the slopes are close to 1 . However the intensity of C-H antisymmetric vibration mode was affected by the $\mathrm{C}-\mathrm{H}$ vibration and the $\mathrm{OH}$ vibration of the carboxylic acids, specifically for Tn-Piv and Tn-Val series. The slope values have thus to be handled carefully. Comparatively to the experiment in water, the slope observed for Tn-Val series was similar than the slope simulated for Hn-Val series, indicating that the THF did not promote the condensation reaction kinetic of one of the condensable molecules.

The FTIR spectra also reveal the presence of the ammonium carboxylate species in samples prepared with carboxylic acid as shown from the Tn-Ac series spectra illustrated in Fig S17. The evolution of $\tau^{\prime}$ values calculated from the equation 2 allows evaluating the contribution of the vibration mode of carboxylate groups $\left(T_{C=O^{-}}\right.$at 1540 $\left.\mathrm{cm}^{-1}\right)$ compared to that of C-H bounds $\left(T_{C-\mathrm{H}^{-}}\right.$at $\left.2920 \mathrm{~cm}^{-1}\right)$.

$\tau^{\prime}=\frac{T_{0}-T_{\mathrm{C}=\mathrm{O}^{-}}}{T_{0}-T_{\mathrm{C}-\mathrm{H}}}$

Fig $13 \mathrm{~b}$ exhibits the trends of $\tau^{\prime}$ as the TEOS increases in the mixture for the Tn-Ac, Tn-Piv and Tn-Val series. The results clearly reveal the decrease of the carboxylate contribution in regards to that of $\mathrm{C}-\mathrm{H}$ bound as the TEOS increases in the mixture for the three considered series. Nevertheless, while the $\tau^{\prime}$ values of the Tn-Ac series are slightly affected by the TEOS addition, those of Tn-Piv and Tn-Val series are drastically reduced. By extrapolating these results, the ratios between AUT and carboxylic acid decrease with the addition of TEOS, in particular for the pivalic and the valproic acid.

The same trend was observed for the Hn-Val series prepared in water (Fig S18), suggesting that the change of solvent is not involved in this phenomenon.

This phenomenon can be linked to the evolution of the surrounding medium as the sol-gel process occurs. The solgel process can be dissociated in two steps, namely the hydrolysis and the condensation. In regular sol-gel process, these two steps usually lead to ethanol coming from the ethoxysilane groups. In a sol-gel process where carboxylic acid performs the catalysis, the mechanism is not very well known. However, it has been demonstrated that the carboxylic acids react with siloxane groups, generating silyl-ester complexes very reactive in regards to other siloxane groups [34]. This study has showed that the carboxylic acid is consumed during this mechanism, leading to ether. Therefore, it is possible that a non-negligible quantity of carboxylic acid stays in solution under silyl-ester complex form or is consumed. The resulting amount of carboxylic acid which can contribute to the formation of ammonium complexes is therefore reduced.

Otherwise, the terbutyl and pentylpropyl groups of the pivalic and valproic acid respectively are electron-donors. The protons of the carboxylic groups are thus less labile compared to that of the acetic acid. The resulting ammonium carboxylate complexes are probably less stable in media containing a large amount of ethanol provided by the aforementioned sol-gel process. In addition, these differences in regards to the donor character also act in the catalytic properties and the stability of the corresponding silyl-ester complex intermediates. Therefore, the 
evolution of the particle size of T5-Ac, T5-Piv and T5-Val has to be compared to their respective ratio $\tau^{\prime}$ featuring the molar ratio between the curvature agent and the AUT molecule into the materials. In addition, the gold Np test shows the presence of amino functions at the surface of hybrid nanoparticles for Tn-Val (Fig S19). We assume that the increase of the particle size results of a less intense confinement of self-assembly around the silica aggregates due to a lower $\tau^{\prime}$ ratio. A reduced confinement offers more volume for the insertion of the silica precursor in the aggregates and lead to larger particles.

\section{Conclusion}

In this study, we attempted to show the morphological versatility offered by this so-called "one pot" synthesis, resulting of combinatory effects of the addition of TEOS and of the role of curvature agent played by the counterion involved during the sol-gel process. Although the curvature agent does not affect drastically the quantitative introduction of silica units in the material, the interactions at the interface governed by the ammonium species affect the shape of aggregates, making of the counter-ion a critical morphological parameter. The morphology of the particles is also closely linked to the immiscibility of TEOS in water. The Fig 14 illustrates different morphologies of silica materials tuned by changes of experimental conditions, such as counter ions, addition of TEOS, and solvents (water or THF), in the sol-gel process using amino-undecyl-triethoxysilane as amphiphilic organosilane. We have shown that it is possible to obtain macroporous materials, blackberry-like and spherical particles, vesicles and nanoparticles whose size can be modulated as a function of the counter-ion. As summarized in Fig. 14, without counter-ion in water we observed macroporous beads when TEOS is added during the sol gel reaction, where connected planar nanoparticles are obtained in THF. In contrast, the ammonium chloride based material led to blackberry-like particles in water which seems to be progressively smoothed by the addition of TEOS. Such a composition in THF exhibits well define nanoparticles. For the materials prepared by using acetic acid as counter-ion, vesicles were obtained with and without TEOS in water instead of nanoparticles in THF. In the meantime, the structural effects of the addition of TEOS appear unequivocal. Indeed, the TEOS addition acts in the self-assembly by incorporating and swelling the structures aside from the counterion of the ammonium species or the solvent. Among the tested compositions, different kinds of aggregates were prepared, and their morphologies are compatible with large accessible surface in the final material. Primary amino group is one of the most attractive surface functionality owing to its use as ligand for various metal ions or as precursor group for post-functionalization. From this chemical versatility combined to the tunability of the structure and the morphology, many applications could ensue in the fields of solid phase extraction or biotechnology for instance.

\section{Acknowledgments}

The authors acknowledge J. Ravaux for assistance on SEM and Wet-STEM experiments, C. Rey and A. Jonchère for their technical support.

\section{References}


(1) Rurack K, Martinez-Manez R (2010) In: The Supramolecular Chemistry of Organic-Inorganic Hybrid Materials, John Wiley \& Sons, Hoboken, New Jersey.

(2) Rosenholm JM, Linden M (2007) Wet-chemical analysis of surface concentration of accessible groups on different amino-functionalized mesoporous SBA-15 silicas. Chemistry of Materials 19 (20):5023-5034.

(3) Wang XG, Lin KSK, Chan JCC, Cheng S (2004) Preparation of ordered large pore SBA-15 silica functionalized with aminopropyl groups through one-pot synthesis. Chemical Communications (23):2762-2763.

(4) Macquarrie DJ, Jackson DB (1997) Aminopropylated MCMs as base catalysts: a comparison with aminopropylated silica. Chemical Communications (18):1781-1782.

(5) Lambert S, Tran KY, Arrachart G, Noville F, Henrist C, Bied C, Moreau JJE, Wong Chi Man M, Heinrichs B (2008) Tailor-made morphologies for $\mathrm{Pd} / \mathrm{SiO} 2$ catalysts through sol-gel process with various silylated ligands. Microporous Mesoporous Mater 115 (3):609-617.

(6) Besnard R, Cambedouzou J, Arrachart G, Le Goff XF, Pellet-Rostaing S (2015) Organosilica-metallic sandwich materials as precursors for palladium and platinum nanoparticle synthesis. RSC Adv 5 (95):7761977628 .

(7) Fryxell GE, Liu J, Hauser TA, Nie ZM, Ferris KF, Mattigod S, Gong ML, Hallen RT (1999) Design and synthesis of selective mesoporous anion traps. Chemistry of Materials 11 (8):2148-2154.

(8) Yokoi T, Yoshitake H, Tatsumi T (2004) Synthesis of amino-functionalized MCM-41 via direct cocondensation and post-synthesis grafting methods using mono-, di- and tri-amino-organoalkoxysilanes. Journal of Materials Chemistry 14 (6):951-957.

(9) Yoshitake H, Koiso E, Horie H, Yoshimura H (2005) Polyamine-functionalized mesoporous silicas: Preparation, structural analysis and oxyanion adsorption. Microporous and Mesoporous Materials 85 (1-2):183194.

(10) Reynhardt JPK, Alper H (2003) Hydroesterification reactions with palladium-complexed PAMAM dendrimers immobilized on silica. Journal of Organic Chemistry 68 (22):8353-8360.

(11) Kim CO, Cho SJ, Park JW (2003) Hyperbranching polymerization of aziridine on silica solid substrates leading to a surface of highly dense reactive amine groups. Journal of Colloid and Interface Science 260 (2):374378.

(12) Lim MH, Stein A (1999) Comparative Studies of Grafting and Direct Syntheses of Inorganic-Organic Hybrid Mesoporous Materials. Chem Mater 11(11): 3285-3295

(13) Arrachart G, Carcel C, Trens P, Moreau JJE, Wong Chi Man M (2009) Silylated Melamine and Cyanuric Acid as Precursors for Imprinted and Hybrid Silica Materials with Molecular Recognition Properties. Chemistry a European Journal 15(25): 6279-6288

(14) Arrachart G, Bendjerriou A, Carcel C, Trens P, Moreau JJE, Wong Chi Man M (2010) Influence of the alkyl linker in the structuring of bridged silsesquioxanes obtained by self-recognition properties New Journal of Chemistry 34(7): 1436-1440.

(15) Arrachart G, Creff G, Wadepohl H, Blanc C, Bonhomme C, Babonneau F, Alonso B, Bantignies J-L, Carcel C, Moreau JJE, Dieudonné P, Sauvajol J-L, Massiot D, Wong Chi Man M (2009) Nanostructuring of hybrid silicas through self-recognition process. Chemistry a European Journal 15(20): 5002-5005

(16) Shimojima A, Sugahara K, Kuroda K (2003) Direct Formation of Mesostructured Silica-Based Hybrids from Novel Siloxane Oligomers with Long Alkyl Chains. Angew. Chem. Int. Ed. 42: 4057-4060

(17) Zhang Q, Ariga K, Okabe A, Aida T (2004) A Condensable Amphiphile with a Cleavable Tail as a "Lizard" Template for the Sol-Gel Synthesis of Functionalized Mesoporous Silica. J Am Chem Soc. 126(4): 988989

(18) Voss R, Thomas A, Antonietti M, Ozin G (2005) A. Synthesis and characterization of highly amine functionalized mesoporous organosilicas by an "“all-in-one"” approach. J. Mater. Chem. 4010-4014.

(19) Ruiz-Hitzky E, Letaïef S, Prévot V (2002) Novel Organic-Inorganic Mesophases: Self-Templating Synthesis and Intratubular Swelling. Adv. Mater. 14: 439-443.

(20) Alauzun J, Besson E, Mehdi A, Reye C, Corriu RJP (2008) Reversible Covalent Chemistry of CO2: An Opportunity for Nano-Structured Hybrid Organic-Inorganic Materials. Chem. Mater. 20(2): 503-513.

(21) Mouawia R, Mehdi A, Reye C, Corriu RJP (2008) From Simple Molecules to Highly Functionalised Lamellar Materials. J. Mater. Chem. 18(17): 2028-2035. 
(22) Besnard R, Arrachart G, Cambedouzou J, Pellet-Rostaing S (2016) Tuning the nanostructure of highly functionalized silica using amphiphilic organosilanes: Curvature agent effects. Langmuir 32(18): 4624-4634.

(23) Besnard R, Cambedouzou J, Arrachart G, Diat O, Pellet-Rostaing S (2013) Self-Assembly of Condensable "Bola-Amphiphiles" in Water/Tetraethoxysilane Mixtures for the Elaboration of Mesostructured Hybrid Materials. Langmuir 29(33): 10368-10375.

(24) Li Y, Beck R, Huang T, Choi MC, Divinagracia M (2008) Scatterless Hybrid Metal-single-Crystal Slit for Small-Angle X-Ray Scattering and High-Resolution X-Ray Diffraction. J. Appl. Crystallogr. 41(6): 11341139.

(25) Pichon BP, Wong Chi Man M, Bied C, Moreau JJE (2005) A Simple Access to $\omega-$ Aminoalkyltrialkoxysilanes: Tunable Linkers for Self-Organised Organosilicas. J Organomet Chem. 1126-1130.

(26) Alauzun J, Mehdi A, Reye C, Corriu RJP (2005) CO2 as a Supramolecular Assembly Agent: A Route for Lamellar Materials with a High Content of Amine Groups. J. Am. Chem. Soc. 127(32): 11204-11205.

(27) Besnard R, Arrachart G, Cambedouzou J, Pellet-Rostaing S (2015) Structural Study of Hybrid Silica Bilayers from "bola-Amphiphile" Organosilane Precursors: Catalytic and Thermal Effects. RSC Adv. 5(71): 57521-57531.

(28) Karlsson S, Friman R, Björkqvist M, Lindström B, Backlund S (2001) Phase Behavior and Characterization of the System Acetic Acid-Dodecylamine-Water. Langmuir 17(12): 3573-3578.

(29) Yang X, Zhao N, Zhou Q, Wang Z, Duan C, Cai C, Zhang X, Xu J (2012) Facile Preparation of Hollow Amino-Functionalized Organosilica Microspheres by a Template-Free Method. J. Mater. Chem. 22(34): 1801018017.

(30) Grabar KC, Freeman RG, Hommer MB, Natan MJ (1995) Preparation and Characterization of Au Colloid Monolayers. Anal. Chem. 67(4): 735-743.

(31) Bacsik Z, Ahlsten N, Ziadi A, Zhao G, Garcia-Bennett AE, Martín-Matute B, Hedin N (2011) Mechanisms and Kinetics for Sorption of $\mathrm{CO} 2$ on Bicontinuous Mesoporous Silica Modified with N-Propylamine. Langmuir 27(17):11118-11128.

(32) Duran A, Serna C, Fornes V, Fernandez Navarro JM (1986) Structural Considerations about SiO2 Glasses Prepared by Sol-Gel. J. Non-Cryst. Solids 82(1-3): 69-77.

(33) Sugahara Y, Inoue T, Kuroda K (1997) 29 Si NMR Study on Co-Hydrolysis Processes in Si(OEt)4$\mathrm{RSi}(\mathrm{OEt}) 3-\mathrm{EtOH}-$ water-HCl Systems (R=Me, Ph): Effect of R groups. J. Mater. Chem. 7(1): 53-59.

(34) Corriu RJP, Nguyên T (2008) In : Chimie Moléculaire, Sol-Gel et Nanomateriaux., Les Editions de l'Ecole Polytechnique, France. 


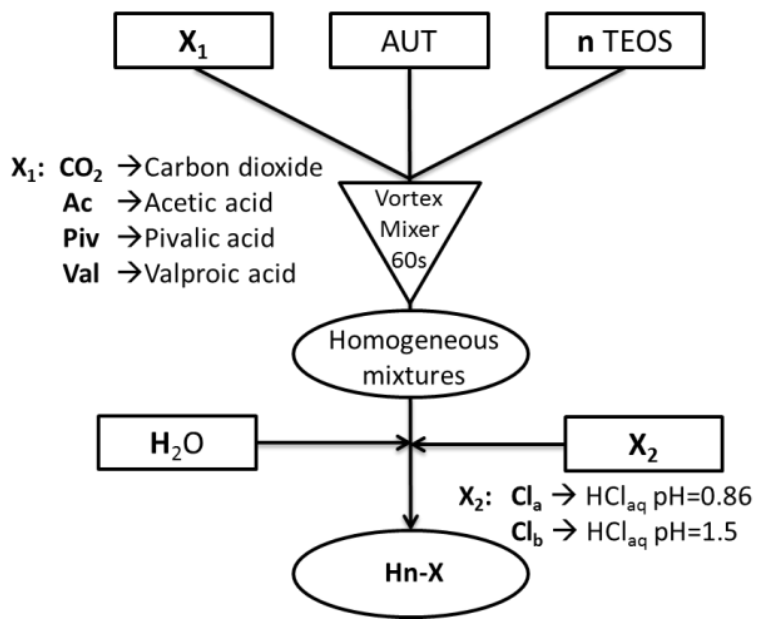

Scheme 1: Methodology for the preparation of the Hn-X series.

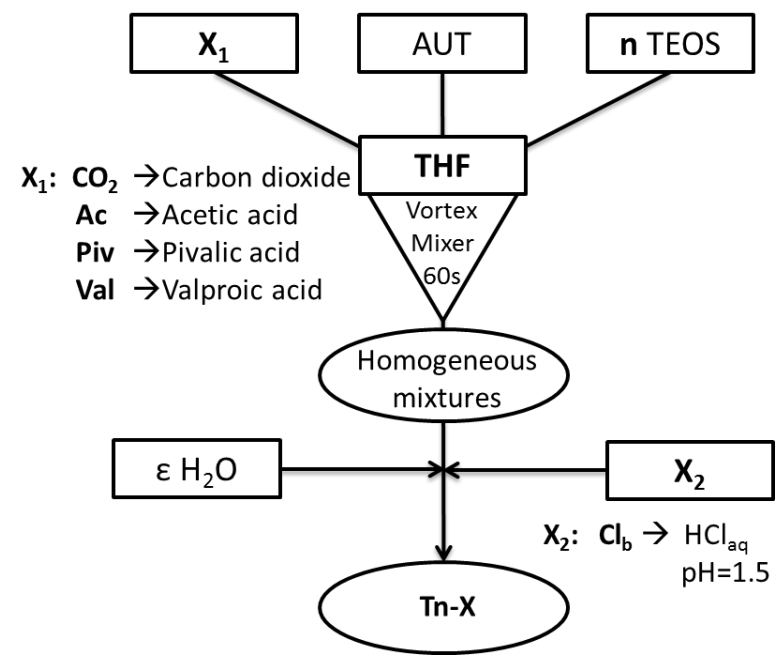

Scheme 2: Methodology for the preparation of the Tn-X series. 
Table 1: Molar ratios of the Hn-X series AUT/TEOS/Catalyst

\begin{tabular}{cccccccc}
\hline AUT/TEOS/ & $\mathrm{Hn}$ & $\mathrm{Hn}-\mathrm{Cl}_{b}$ & $\mathrm{Hn}-\mathrm{CO}_{2}$ & $\mathrm{Hn}-\mathrm{Cl}_{\boldsymbol{a}}$ & $\mathrm{Hn}-\mathrm{Ac}$ & $\mathrm{Hn}-\mathrm{Piv}$ & $\mathrm{Hn}-\mathrm{Val}$ \\
\hline $\mathbf{1 / 0} /$ & 0 & 0.3 & 0.3 & 1.4 & 1 & 1 & 1 \\
$\mathbf{1 / 0 . 5 /}$ & 0 & 0.4 & 0.4 & 1.8 & - & - & - \\
$\mathbf{1 / 1} /$ & 0 & 0.5 & 0.5 & 2.2 & 1.6 & 1.6 & 1.6 \\
$\mathbf{1 / 1 . 5 /}$ & 0 & 0.6 & 0.6 & 2.65 & - & - & - \\
$\mathbf{1 / 5} /$ & 0 & 1.3 & 1.3 & 5.6 & 4.1 & 4.1 & 4.1 \\
$\mathbf{1 / 1 0} /$ & 0 & 2.3 & 2.3 & 10 & - & - & - \\
\hline
\end{tabular}

Ligh grey : basic condition ; Dark grey : acidic condition

Table 2: Molar ratios of the $\mathrm{Tn}$-X series AUT/TEOS/ $\mathrm{H}_{2} \mathrm{O} /$ Catalyst

\begin{tabular}{cccccc}
\hline AUT/TEOS/H & Tn $/$ & $\begin{array}{c}\text { Tn-CO } \\
\text { Tn-Cl }\end{array}$ & Tn-Ac & Tn-Piv & Tn-Val \\
\hline $\mathbf{1 / 0 / 1 . 5 /}$ & 0 & 0.001 & 1 & 1 & 1 \\
$\mathbf{1 / 1 / 3 . 5 /}$ & 0 & 0.002 & 1.6 & 1.6 & 1.6 \\
$\mathbf{1 / 5 / 1 1 . 5 /}$ & 0 & 0.006 & 4.1 & 4.1 & 4.1 \\
\hline
\end{tabular}

Ligh grey : basic condition 


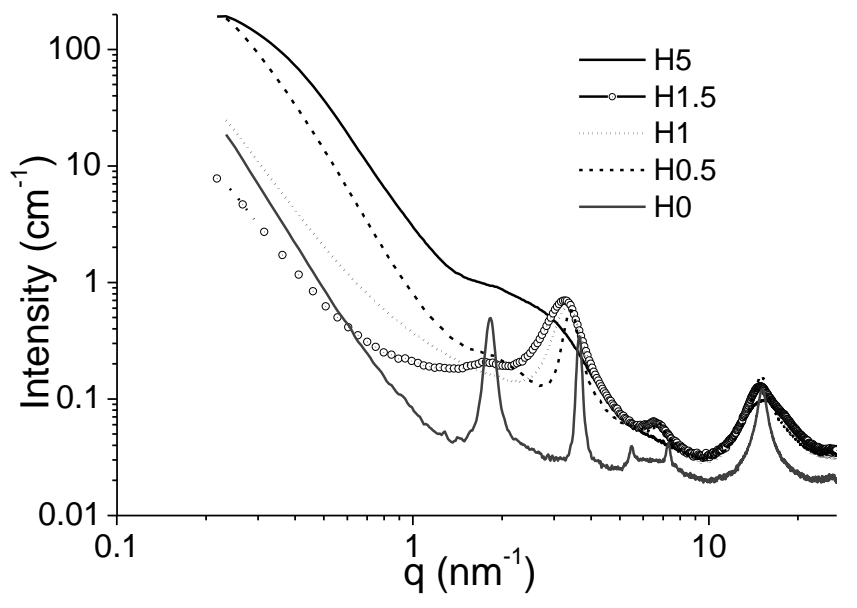

Fig. 1 SAXS profile of samples H0 (grey straight line), H0.5 (dashes), H1 (dots), H1.5 (rings) and H5 (black straight line).

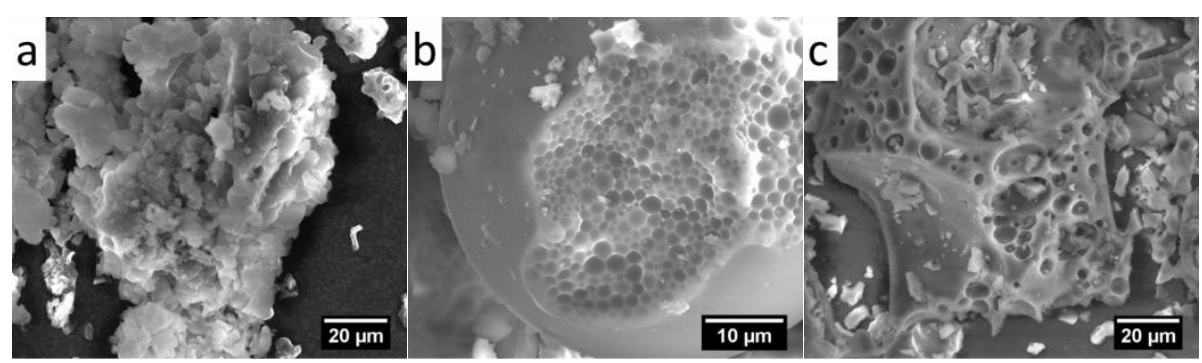

Fig. 2 SEM images of samples a) H0, b) H1 and c) H5.

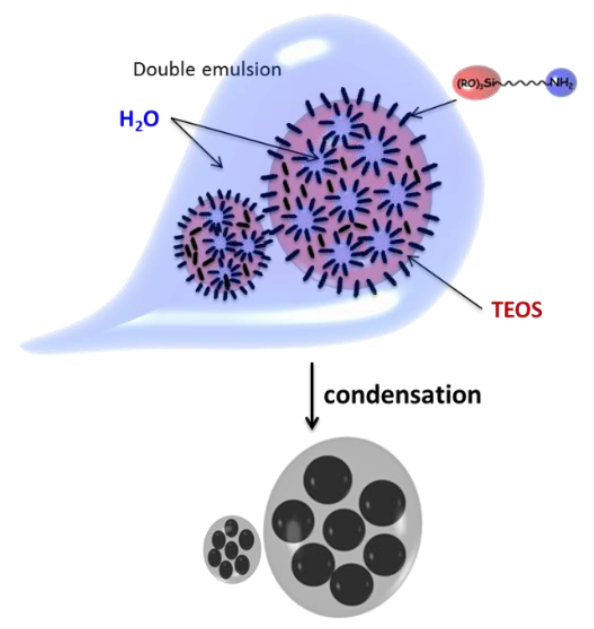


Fig. 3 Schematic representation of the formation mechanism of macroporous beads coming from double emulsion of water droplets trapped in TEOS droplets in water.
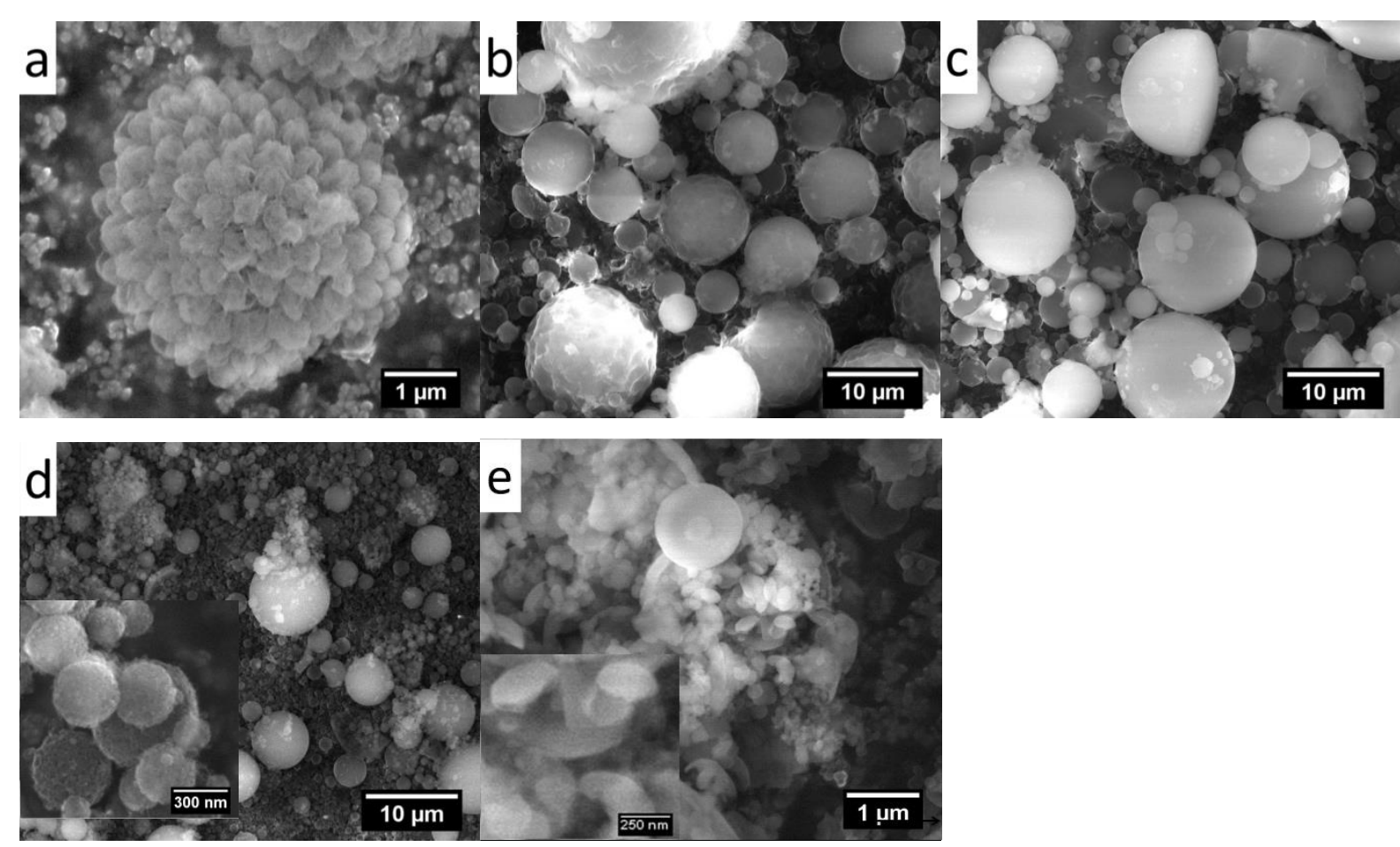

Fig. 4 SEM images of materials a) $\mathrm{H} 0-\mathrm{Cl}$, b) $\mathrm{H} 0.5-\mathrm{Cl}_{b}$, c) $\mathrm{H} 1-\mathrm{Cl}_{b}$, d) $\mathrm{H} 5-\mathrm{Cl}_{\mathrm{b}}$ and e) $\mathrm{H} 10-\mathrm{Cl}_{\mathrm{b}}$.

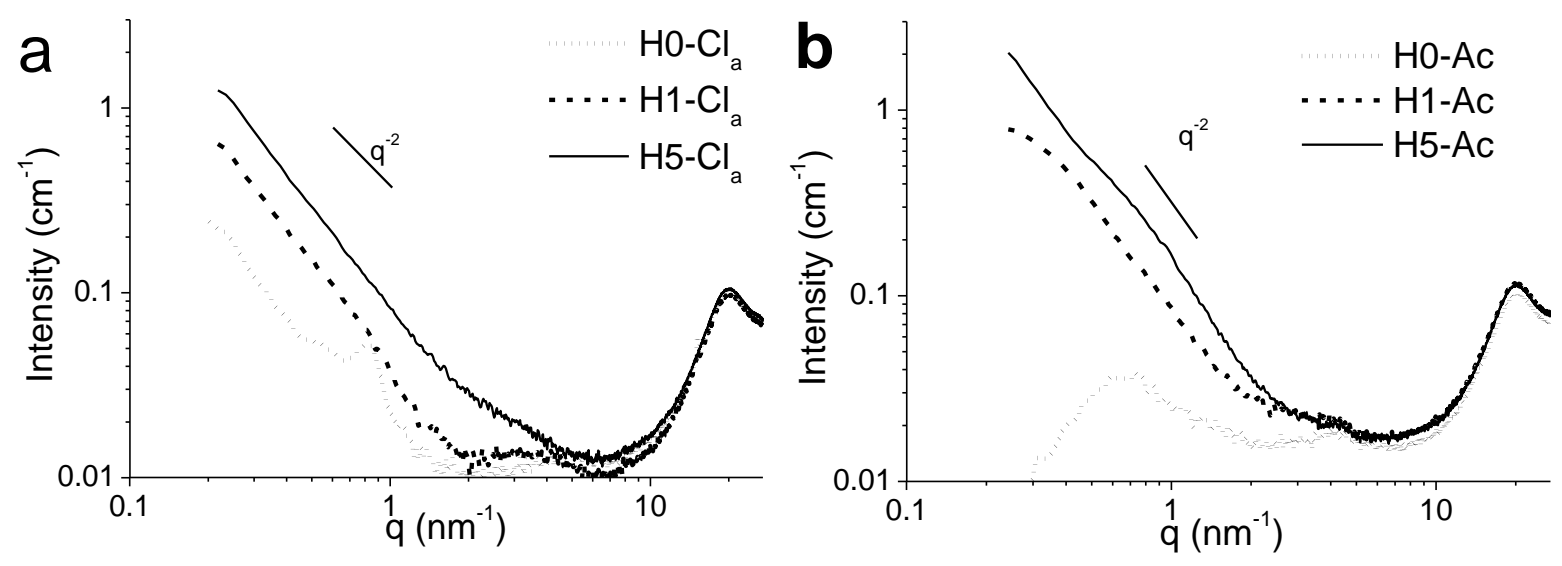

Fig. 5 SAXS profiles of a) $\mathrm{H} 0-\mathrm{Cl}_{\mathrm{a}}, \mathrm{H} 1-\mathrm{Cl}_{\mathrm{a}}$, and $\mathrm{H} 5-\mathrm{Cl}_{\mathrm{a}}$; b) $\mathrm{H} 0-\mathrm{Ac}, \mathrm{H} 1-\mathrm{Ac}$, and H5- Ac. 


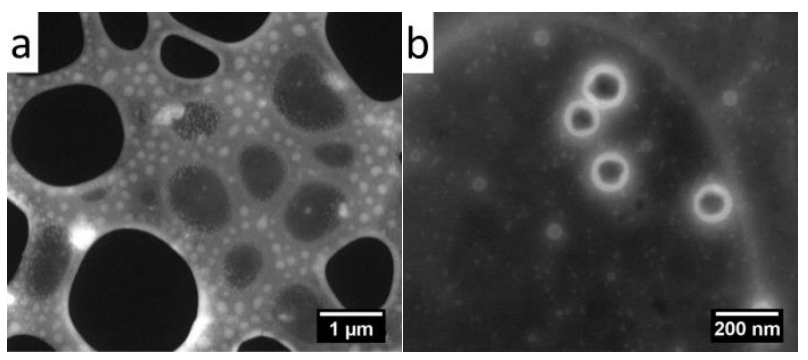

Fig. 6 Wet STEM images of suspensions a) H5-Cla and b) H5-Ac.
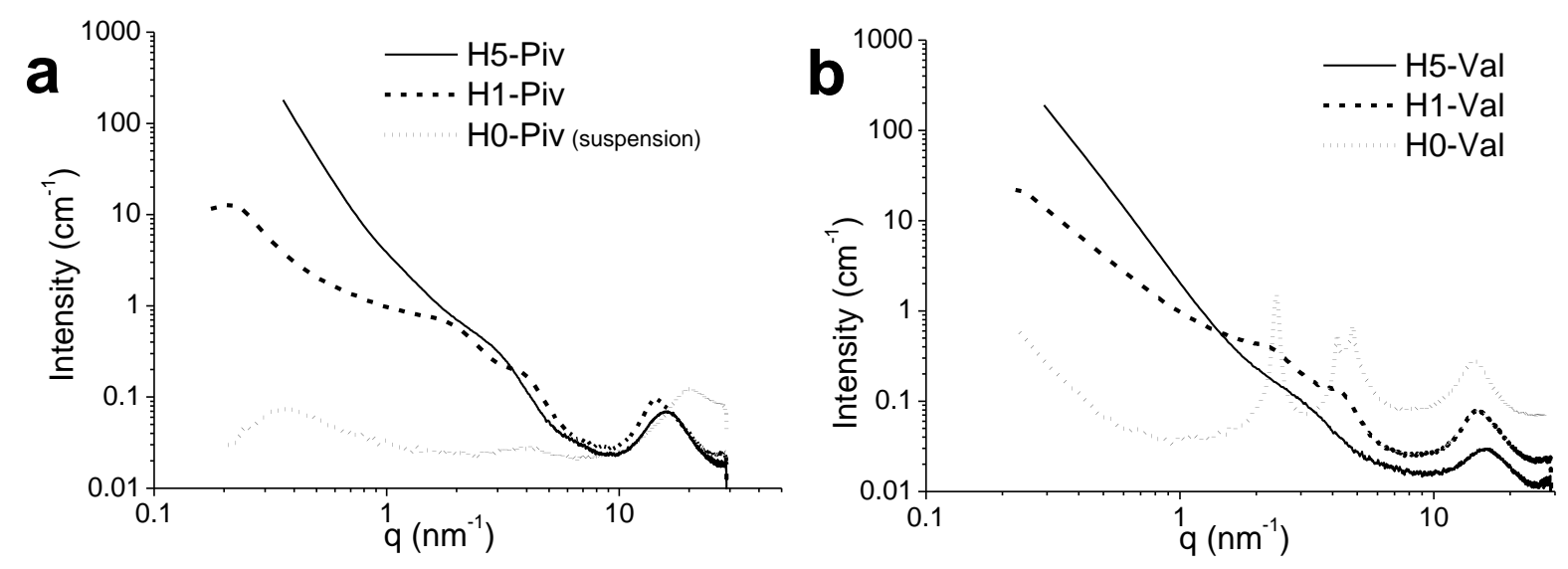

Fig. 7 SAXS profiles of a) H0-Piv, H1-Piv, and H5-Piv; b) H0-Val, H1-Val, and H5-Val.

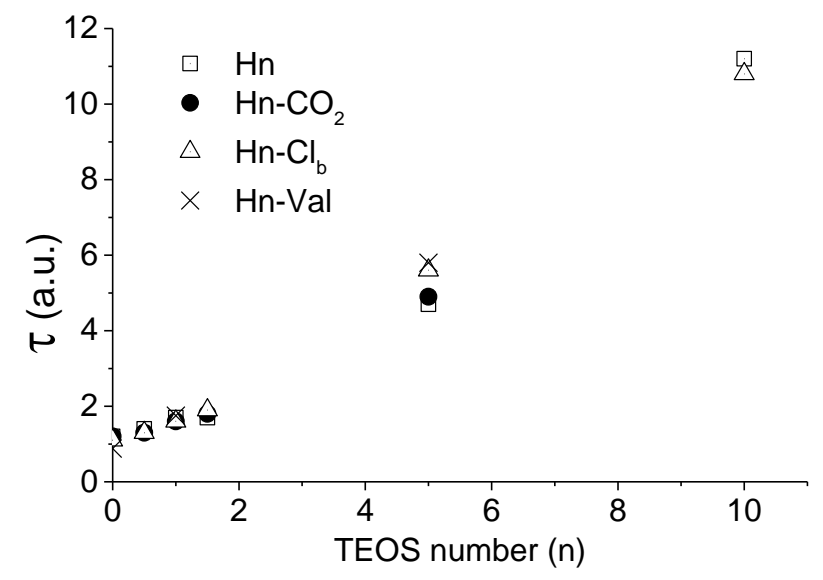

Fig. 8 Evolution of $\tau$ as function of the TEOS number introduced in the initial mixture for the materials of the $\mathrm{Hn}, \mathrm{Hn}-\mathrm{CO}_{2}, \mathrm{Hn}-\mathrm{Cl}_{\mathrm{b}}$, and $\mathrm{Hn}-\mathrm{Val}$ series. 


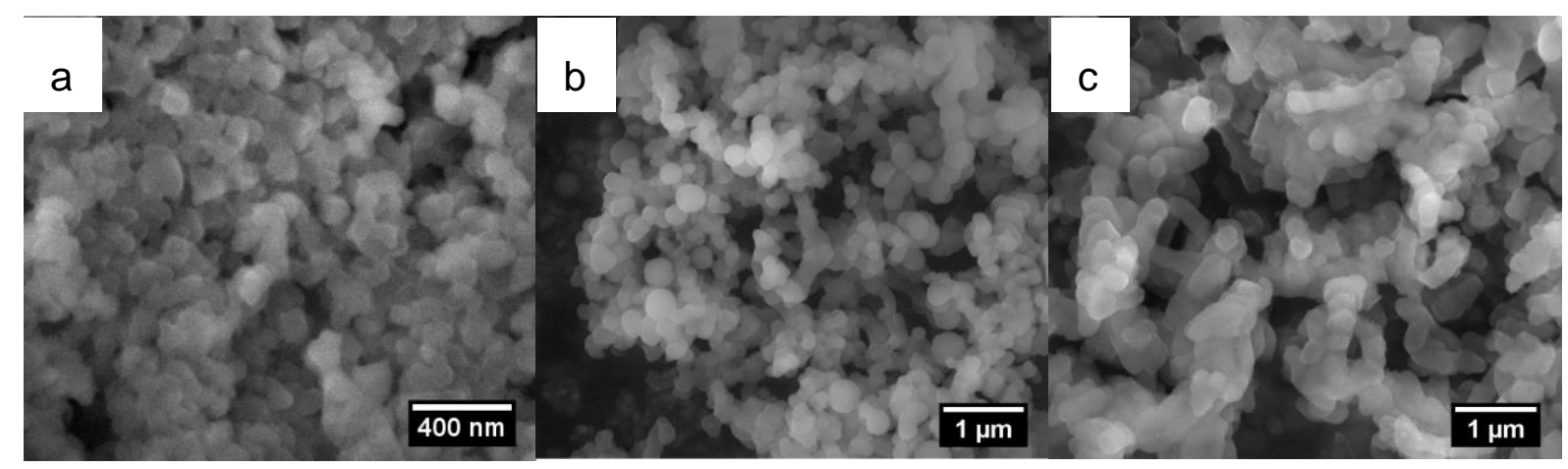

Fig. 9 SEM images of a) T0, b) T1 and c) T5 materials.
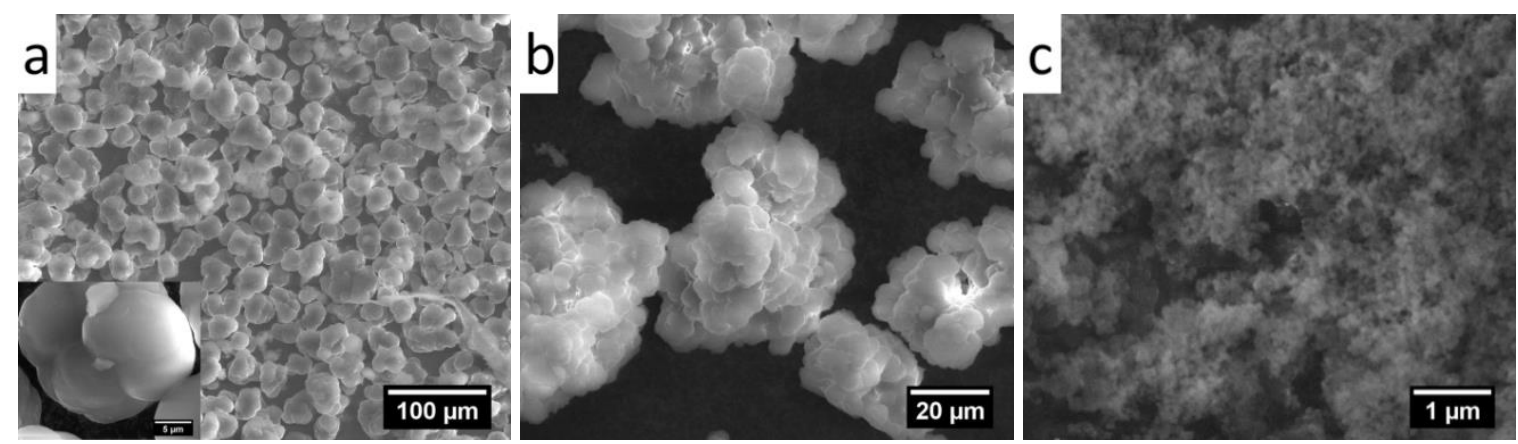

Fig. 10 SEM images of a) T0-CO

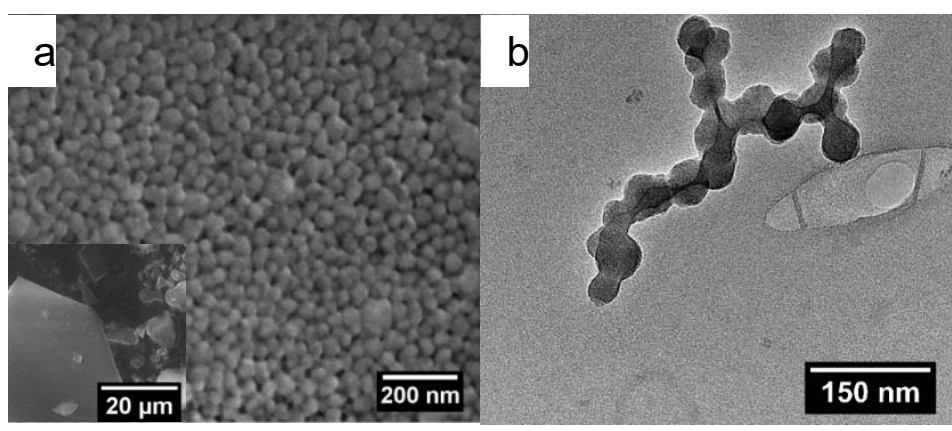

Fig. 11 a) SEM images of T5-Cl $\mathrm{b}_{\mathrm{b}}$, b) TEM image of T5-Cl $\mathrm{l}_{\mathrm{b}}$. 


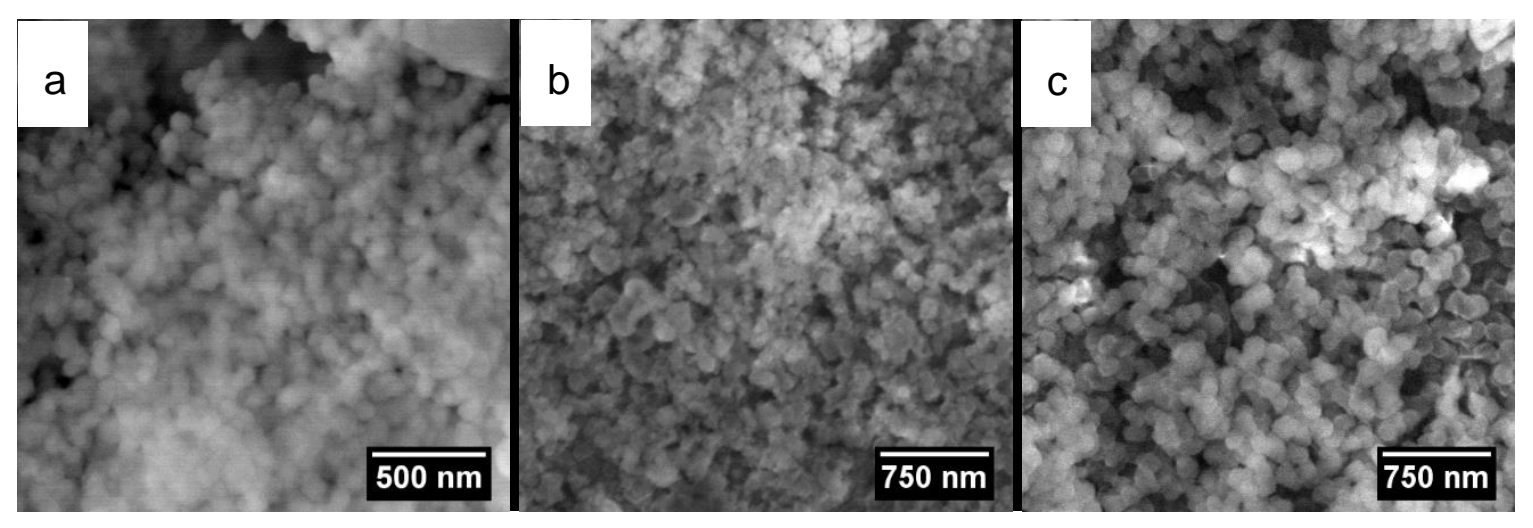

Fig. 12 SEM images of a) T5-Ac, b) T5-Piv and c) T5-Val materials.
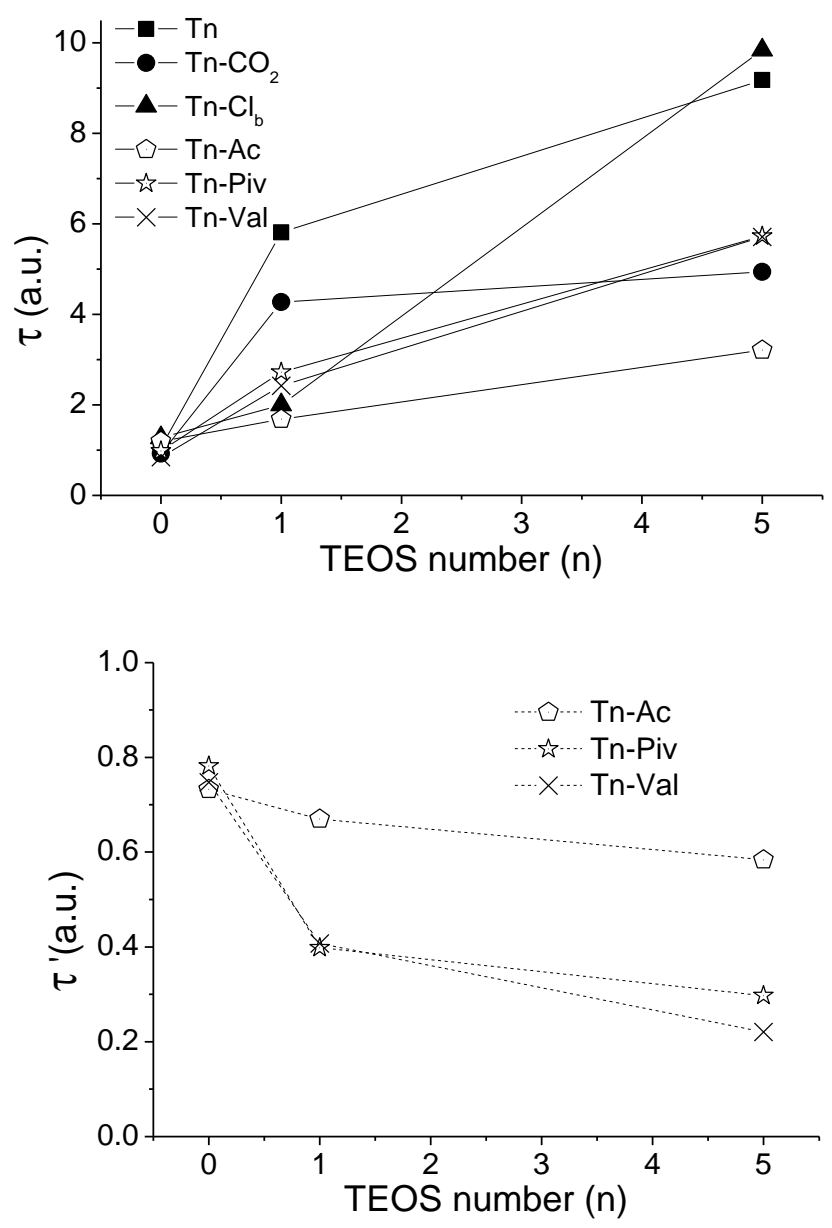
Fig. 13 a) Evolution of $\tau$ as a function of the TEOS number introduced in the initial mixtures for the materials of the Tn-X series; b) Evolution of $\tau^{\prime}$ as function of the added TEOS number in the initial mixtures for the materials of the Tn-X series (X= Ac, Piv and Val).

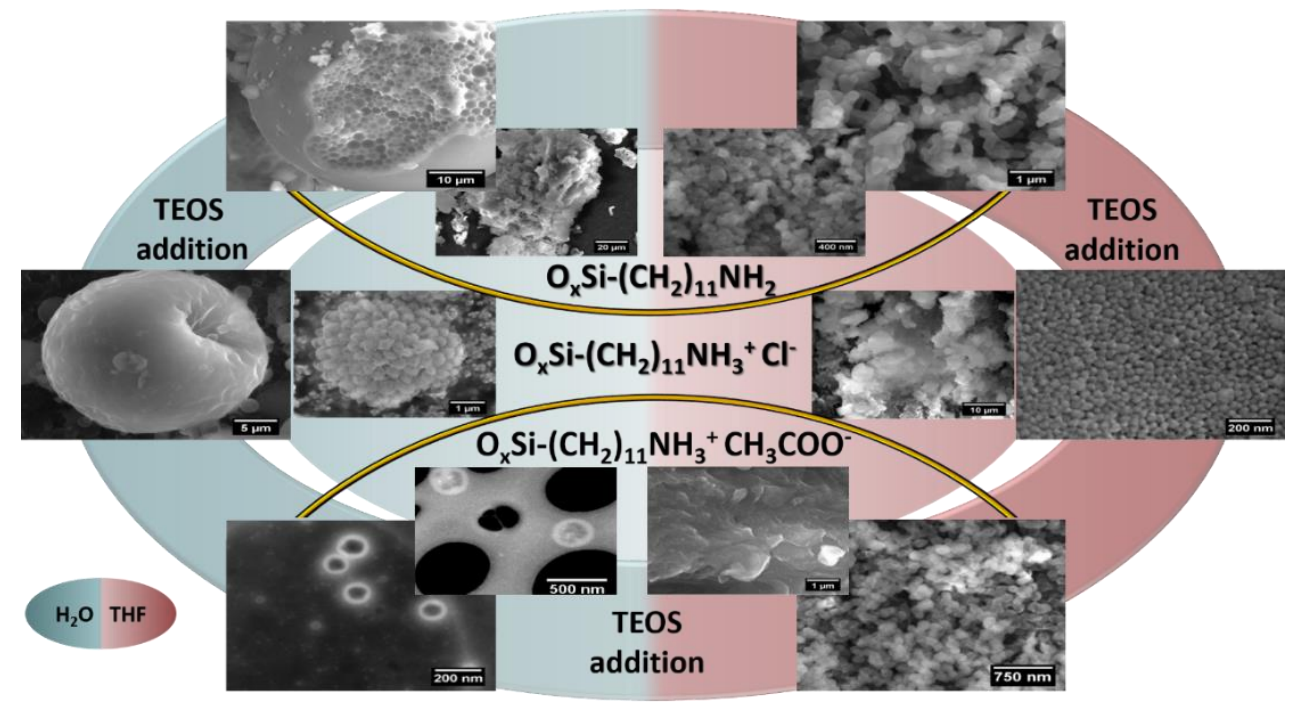

Fig. 14 Schematic representation summarizing the morphological effects of TEOS addition depending on the solvent and the counter-ion associated to the amino function of the AUT molecule. 